\title{
MAN_03 - Technology foresight for identification of opportunities and partnerships in COVID-19 vaccines, biotherapeutics and diagnostics test
}

Diana Praia Borges Freire ${ }^{*}$; Christiane de Fátima Silva Marques ${ }^{1}$; Lívia Rubatino de Faria ${ }^{1}$; Camila Faria Magalhães ${ }^{1}$; Ana Rodrigues de Andrade ${ }^{1}$; Ana Carolina dos Reis Albuquerque Cajaraville ${ }^{1}$; Renata Tourinho Santos ${ }^{1}$; Gisela Freitas Trindade ${ }^{1}$; Priscila do Nascimento Silva ${ }^{1}$; Beatriz de Castro Fialho ${ }^{1}$. ${ }^{1}$ Fiocruz/Bio-Manguinhos.

Introduction: Bio-Manguinhos has been establishing systematic Technology Foresight (TF), based on the experience acquired in TF focused on plant platform, later in 2018, as one of the strategies for tackling the COVID-19 pandemic. TF is an ongoing process that aims to map projects and products under development for COVID-19 (vaccines, biopharmaceuticals and diagnostics) in a worldwide framework.

Objective: To present the TF initiatives regarding the design of the process and its use as a tool to analyze the current COVID-19 scenario.

Methodology: To establish and design a TF process, the starting research questions were: "which are the products under development for COVID-19", "what are their development status" and "which organizations are involved". To address those questions, a multidisciplinary network was established in Bio-Manguinhos with Foresight Groups (FG), Discussion Groups (DG) and Opportunities Group (OG). Given the specificities of each segment, three subgroups were created for vaccines, biopharmaceuticals, and diagnostics, with FG, DG e OG participants. The FG collected data from different databases and websites to identify product pipelines, projects, clinical studies, technologies, patents, and scientific publications (including articles, preprints, newsletter and press releases). For data preparation, the FG set up different data transformation strategies, creating proprietary data tables. For analysis and visualization, the FG used data mining and descriptive statistics in Microsoft Excel ${ }^{\circledR}$ and Power BI ${ }^{\circledR}$. In parallel, to improve data enrichment, the FG and DG worked closely to discuss and study scientific publications and clinical trials and interacted with $O G$ to streamline scenario analyzes to subsidize negotiations and partnerships.

Results: The result of this work is three-fold: agile scenario building and analysis to support decision making regarding COVID-19 product partnerships; improvement of a previous foresight process used for plant-based technologies; the development of a structured data tables covering more than 900 development initiatives in the three Bio-Manguinhos product lines since the beginning of COVID-19 pandemic. The data enrichment applied during the treatment step allowed to analyze product and technological platforms, technology details and respective development phases, geographical localization of R\&D efforts, networks of collaboration, players, and market positioning strategies.

Conclusion: Besides contributing to support and improve Bio-Manguinhos technological decisions regarding COVID-19, as an integral part of the ongoing efforts has also improved technological decision making based on foresight approaches. The methodological improvement and additional techniques adopted to address the COVID-19 pandemics hasn't only contributed to consolidate the knowledge accumulated in previous foresight initiatives but has also stimulated the design of a proprietary database, whose conceptual model is tailored to the different kind of data and information. Besides demonstrating the importance of institutionalizing foresight practices, the establishment of a multidisciplinary and multilevel collaborative work contributed to improve the quality of scenario building as well as the generation and dissemination of institutional knowledge across different areas.

Keywords: Technology foresight; COVID-19 pandemic; Scenario analyzes 